\title{
THE BASS-QUILLEN CONJECTURE AND SWAN'S QUESTION
}

\author{
DORIN POPESCU
}

\begin{abstract}
We present a question which implies a complete positive answer for the Bass-Quillen Conjecture.

Key words : Regular Rings, Smooth Morphisms, Projective Modules 2010 Mathematics Subject Classification: Primary 13C10, Secondary 19A13,13H05,13B40.
\end{abstract}

\section{INTRODUCTION}

The theory of projective modules over polynomial algebras over a regular ring $R$ was an important subject in Commutative Algebra starting with Serre's Conjecture (see [2]) and its extension considered by Bass and Quillen.

Conjecture 1. (Bass-Quillen Conjecture, [1, Problem IX], 9]) Let $R$ be a regular ring. Every finitely generated projective module $P$ over a polynomial $R$-algebra, $R[T], T=\left(T_{1}, \ldots, T_{n}\right)$ is extended from R, i.e. $P \cong R[T] \otimes_{R}(P /(T) P)$.

Important positive answers were given by Quillen (see [9]) and Suslin (see [13]) in dimension $\leq 1$ and Murthy [5] in dimension 2. Later Lindel [3] and Swan [6] gave, in particular, positive answers for many regular local rings, essentially of finite type Z-algebras.

Theorem 2. (Lindel, Swan) Let $(R, \mathfrak{m}, k)$ be a regular local ring, essentially of finite type over $\mathbf{Z}$ and $p=$ char $k$. The following statements hold.

(1) If $p=0$ then $R$ is essentially smooth over its prime field.

(2) If $p \notin \mathfrak{m}^{2}$ then $R$ is essentially smooth over $\mathbf{Z}$.

(3) If $p=0$ or $p \notin \mathfrak{m}^{2}$ then the BQ Conjecture holds for $R$.

Then Swan noticed that it is useful to have a positive answer to the following question.

Question 3. (Swan [6]) Is it a regular local ring a filtered inductive limit of regular local rings, essentially of finite type over $\mathbf{Z}$ ?

The Bass-Quillen Conjecture (shortly BQ Conjecture) is connected with the following one.

Conjecture 4. (Bass-Quillen-Suslin Conjecture) Let $R$ be a local ring, Assume either that $R$ is regular local ring or that $1 / r ! \in R$. Let $v$ be a unimodular vector over $R[T]$ of length $(r+1)$. Then $v$ can be completed to an invertible matrix over $R[T]$.

This conjecture holds in many cases given for example by Rao [10, [11 who also proved the BQ Conjecture for regular local rings of dimension 3 with residue characteristic $\neq 2,3$. 
For a regular local ring $(R, \mathfrak{m}, k)$ containing a field, or with $p:=$ char $k \notin \mathfrak{m}^{2}$, Swan's question has a positive answer in [7]. Using this partial positive answer and (iii) from Theorem 2 we got the following corollary (see [7, Theorem 4.1] and also [14, Theorems 2.1, 2.2]).

Corollary 5. The Bass-Quillen Conjecture holds for $R$ if $p=0$ or $p \notin \mathfrak{m}^{2}$.

Recently, we gave a complete positive answer to Swan's Question in [8, Theorem 17] (see here Theorem 7).

The purpose of this paper is to show that a positive answer to the following question gives a complete positive answer to the BQ Conjecture (see Theorem 12).

Question 6. Let $(R, \mathfrak{m})$ be a regular local ring, which is essentially smooth over $\mathbf{Z}_{(p)}$ and $b \in \mathfrak{m}^{2}$. Is it true the $B Q$ Conjecture for the regular local ring $R /(p-b)$ ?

We owe thanks to Ravi Rao for some useful comments.

\section{The Bass-Quillen Conjecture}

We start reminding some definitions concerning smooth morphisms after [4], or [14]. A ring morphism $R \rightarrow R^{\prime}$ of Noetherian rings has regular fibers if for all prime ideals $\mathfrak{p} \in \operatorname{Spec} R$ the ring $R^{\prime} / \mathfrak{p} R^{\prime}$ is a regular ring. It has geometrically regular fibers if for all prime ideals $\mathfrak{p} \in \operatorname{Spec} R$ and all finite field extensions $K$ of the fraction field of $R / \mathfrak{p}$ the ring $K \otimes_{R / \mathfrak{p}} R^{\prime} / \mathfrak{p} R^{\prime}$ is regular. A flat morphism of Noetherian rings is regular if its fibers are geometrically regular. If it is regular of finite type, or essentially of finite type then it is called smooth, resp. essentially smooth.

The proof of our positive answer to Swan's Question says actually a little more (see [8, Theorem 17]).

Theorem 7. Every regular local ring $(R, \mathfrak{m}, k)$ with $0 \neq p=$ char $k \in \mathfrak{m}^{2}$ is a filtered inductive limit of regular local rings $R_{i}$, essentially smooth over a regular local $\mathbf{Z}$ algebra $A_{i} /\left(p-b_{i}\right)$, where $\left(A_{i}, \mathfrak{a}_{i}\right)$ is a regular local ring, essentially smooth over $\mathbf{Z}_{(p)}$, and $b_{i} \in \mathfrak{a}_{i}^{2}$.

In fact, this theorem gives also the structure of regular local rings essentially of finite type over $\mathbf{Z}$ in the case $0 \neq$ char $k \in \mathfrak{m}^{2}$, which is not covered by (i),(ii) from Theorem 2 .

Corollary 8. Let $B$ be a $\mathbf{Z}$-algebra regular local, essentially of finite type. Then $B$ has the form $B=A /(p-b)$, where $(A, \mathfrak{a})$ is a regular local ring essentially smooth over $\mathbf{Z}$ and $b \in \mathfrak{a}^{2}$.

Proof. Applying Theorem 7 to $B$ we see that there exists a regular local $\operatorname{ring} D$, essentially smooth over a regular local $\mathbf{Z}$-algebra $A /(p-b)$, where $(A, \mathfrak{a})$ is a regular local ring, essentially smooth over $\mathbf{Z}_{(p)}$, and $b \in \mathfrak{a}^{2}$ such that the identity of $B$ factors through $D$. Then $B \cong D / q$ for some prime ideal $q \subset D$. Unfortunately, we cannot conclude that $B$ is among these $D$ (see the next remark).

Note that $D$ is a factor of an essentially smooth $\mathbf{Z}_{(p)}$-algebra $D^{\prime}$ by $\left(p-b^{\prime}\right)$, where $b^{\prime}$ is a lifting of $b$ to $D^{\prime}$. Let $q^{\prime}$ be the prime ideal of $D^{\prime}$ containing $p-b^{\prime}$ and such 
that $q^{\prime} /\left(p-b^{\prime}\right)=q$. Changing $D$ by $D^{\prime}$ and $q$ by $q^{\prime}$ we may assume that $D$ is essentially smooth over $\mathbf{Z}_{(p)}$. Then $D$ is an etale neighborhood of a localization of a polynomial algebra in $t$ variables $Y$ over $\mathbf{Z}_{(p)}$ (see e.g. [14, Theorem 2.5]) and $(p-b, Y)$ generates the maximal ideal of $D$. Since $D, B$ are regular local we see that $q$ is generated by a part of a regular system of parameters of $D$, let us say $p-b, z$, where $z=\left(z_{1}, \ldots, z_{r}\right), r \leq t$. After some linear transformations on $Y$ we may assume that $z_{i}=Y_{i}, 1 \leq i \leq r$. Then $B=D / q$ is an etale neighborhood of a localization of $\mathbf{Z}_{(p)}\left[Y_{r+1}, \ldots, Y_{t}\right] /\left(p-b^{\prime \prime}\right), b^{\prime \prime}$ being induced by $b$.

Remark 9. Let $B$ be a regular local ring essentially of finite type over $\mathbf{Z}, D_{n}=$ $B\left[X_{n}\right], n \in \mathbf{N}$ and $\varphi_{n, n+1}: D_{n} \rightarrow D_{n+1}$ be the $B$-morphism given by $X_{n} \mapsto 0$. Then $B$ is the limit of $\left(D_{n}, \varphi_{n, n+1}\right)$, the inclusion $B \subset D_{n}$ has a retraction and $B \approx D_{n}$ for any $n \in \mathbf{N}$.

Next we will need the following two lemmas, the first one is elementary (see e. g. [7. Theorem 4.2]).

Lemma 10. Let $R$ be a regular local ring, which is a filtered inductive limit of some regular local rings $\left(R_{i}\right)_{i \in I}$. If the $B Q$ Conjecture holds for all $R_{i}, i \in I$, then it holds for $R$ too.

Proof. Let $M$ be a finitely generated projective module over $R[T], T=\left(T_{1}, \ldots, T_{n}\right)$. Then $M \cong R \otimes_{R_{i}} M_{i}$ for some finitely generated projective $R_{i}[T]$-module $M_{i}$. Indeed, if $M$ is defined by an idempotent $\varphi$ from $\operatorname{End}(L)$ for some $L=R^{t}$ then we may find $i$ such that $\varphi$ is extended from an endomorphism $\varphi_{i}$ of $R_{i}^{t}$. Also we may find $i$ such that $\varphi_{i}$ is idempotent, and defines the wanted $M_{i}$. As BQ Conjecture holds for $R_{i}$ we get $M_{i}$ free and so $M$ is free too.

The following lemma follows easily from [3]. However, we give here a proof in sketch.

Lemma 11. Let $R \rightarrow R^{\prime}$ be an essentially smooth morphism between regular local rings. If the $B Q$ Conjecture holds for $R$ then it holds for $R^{\prime}$ too.

Proof. $R^{\prime}$ is an etale neighborhood of a localization of a polynomial algebra $A$ over $R$ (see e.g. [14, Theorem 2.5]). If the BQ Conjecture holds for $R$ then it holds for $A$ too by [12. Now it is enough to apply the Corollary from [3].

Theorem 12. If Question [6 has a positive answer then the BQ Conjecture holds for all regular rings.

Proof. By Quillen's Patching Theorem [9, Theorem 1] we may prove the conjecture only for regular local rings. Let $(R, \mathfrak{m}, k)$ be a regular local ring. Using [7, Theorem 3.1] we may suppose that $0 \neq p:=\operatorname{char} k \in \mathfrak{m}^{2}$.

By Theorem [7, $R$ is a filtered inductive limit of some regular local rings $D$, essentially smooth over a regular local ring of the form $A /(p-b)$, where $(A, \mathfrak{a})$ is a regular local ring, essentially smooth over $\mathbf{Z}$ and $b \in \mathfrak{a}^{2}$.

Then the BQ Conjecture holds for $A$ by Lemma 11 and for $A /(p-b)$ by Question 6 . Applying Lemma 11 it follows that BQ holds for $D$. The final result is a consequence of Lemma 10. 
We end the section with a special form of Theorem 7 in the frame of the discrete valuation rings (DVRs for short) in the idea of [8, Theorem 8]. Actually, [8, Theorem 8] has a complicated proof given to illustrate [8, Theorem 17] in the DVR case. The proof below is easier and does not use Néron Desingularization.

Theorem 13. Let $(A, \mathfrak{m}, k)$ be a DVR with $0 \neq p=$ char $k \in \mathfrak{m}^{2}$. Suppose that $k$ is separably generated over $\mathbf{F}_{p}$. Then $A$ is a filtered inductive union of DVRs essentially of finite type over $\mathbf{Z}$.

Proof. As in [8, Theorem 8], let $y=\left(y_{i}\right)_{i \in I}$ be a system of elements of $A$ inducing a separable transcendence base $(\bar{y})$ of $k$ over $\mathbf{F}_{p}$. Then $C_{0}=\mathbf{Z}[Y]_{p} \mathbf{Z}[Y]$ for some variables $Y=\left(Y_{i}\right)_{i \in I}$ is a DVR and the map $C_{0} \rightarrow A, Y \rightarrow y$ defines a ramified extension inducing an algebraic separable residue field extension $\mathbf{F}_{p}(\bar{y}) \subset k$.

Note that $A$ is a filtered inductive union of DVRs $A_{L}=A \cap L$ with $L \subset \operatorname{Fr}(A)$ a finite type field extension of $\mathbf{Q}(y)$. We claim that $L$ must be finite over $\mathbf{Q}(y)$. Indeed, assume that $z \in L$ is transcendental over $\mathbf{Q}(y)$ and $\mathfrak{m}^{s} \subset A$ for some $s \in \mathbf{N}$. Choose $r$ such that $p^{r}>s$ and consider the DVR extension $A_{L^{\prime \prime}} \subset A_{L^{\prime}}$ for $L^{\prime}=\mathbf{Q}(y, z)$ and $L^{\prime \prime}=\mathbf{Q}\left(y, z^{p^{r}}\right)$. The residue field extension induced by $A_{L^{\prime \prime}} \subset A_{L^{\prime}}$ is pure inseparable and also separable by assumption. Then it is trivial and so the ramification index of $A_{L^{\prime \prime}} \subset A_{L^{\prime}}$ is $p^{r}>s$. Contradiction!

Since $L$ is finite over $\mathbf{Q}(y)$ it follows that $A_{L}$ is a localization of the integral closure of $C_{0}$ in $L$ and so essentially finite over $C_{0}$, which is enough.

\section{SWAN'S QUESTION IN THE NON-REDUCED CASE}

We start this section reminding the first part of [8, Theorem 17].

Theorem 14. Let $(A, \mathfrak{m}, k)$ be a Noetherian local ring, $s=\left(s_{1}, \ldots, s_{m}\right)$ some positive integers and $\gamma=\left(\gamma_{1}, \ldots, \gamma_{m}\right)$ a system of nilpotents of $A$. Suppose that $0 \neq p \in \mathfrak{m}^{2}, R=A /(\gamma)$ is a regular local ring and $A$ is a flat $\mathfrak{N}=\mathbf{Z}_{(p)}[\Gamma] /\left(\Gamma^{s}\right)$ algebra, $\Gamma \mapsto \gamma$ with $\Gamma=\left(\Gamma_{1}, \ldots, \Gamma_{m}\right)$ some variables, and $\left(\Gamma^{s}\right)$ denotes the ideal $\left(\Gamma_{1}^{s_{1}} \cdots \Gamma_{m}^{s_{m}}\right)$. Then $A$ is a filtered inductive limit of some Noetherian local $\mathfrak{N}$-algebras $\left(F_{i}\right)_{i}$ essentially of finite type with $F_{i} / \Gamma F_{i}$ regular local rings.

Remark 15. The above theorem holds also when $p=0$ or $p \notin \mathfrak{m}^{2}$ as in $[7$, Theorem 4.1] because the map $\mathfrak{N} \rightarrow A$ is regular in this case.

Lemma 16. In the notation and hypothesis of the above theorem, let $(B, \mathbf{b})$ be a Noetherian local ring, and $z=\left(z_{1}, \ldots, z_{m}\right)$ a system of elements of $B$. Suppose that

(1) for all $i \in[m] s_{i}>1, z_{i}^{s_{i}}=0$,

(2) for all $i \in[m]$ and $j \in\left[s_{i}-1\right],\left(z_{1}, \ldots, z_{i-1}\right): z_{i}^{j}=\left(z_{1}, \ldots, z_{i-1}, z_{i}^{s_{i}-j}\right)$,

(3) $B /(z)$ is a regular local ring.

Then $B$ is a flat $\mathfrak{N}$-algebra by the $\operatorname{map} \varphi: \mathfrak{N} \rightarrow B, \Gamma \mapsto z$.

Proof. Suppose $m=1$. Note that the minimal free resolution of $\mathfrak{N} /(\Gamma)$,

$$
\ldots \rightarrow \mathfrak{N} /\left(\Gamma^{s}\right) \stackrel{\Gamma}{\rightarrow} \mathfrak{N} /\left(\Gamma^{s}\right) \stackrel{\Gamma^{s-1}}{\rightarrow} \mathfrak{N} /\left(\Gamma^{s}\right) \stackrel{\Gamma}{\rightarrow} \mathfrak{N} /\left(\Gamma^{s}\right) \rightarrow \mathfrak{N} /(\Gamma) \rightarrow 0
$$


gives after tensorizing with $B$ a minimal free resolution of $B /(z)$. Thus

$\operatorname{Tor}_{1}^{\mathfrak{N}}(\mathfrak{N} /(\Gamma), B)=0$. As $B /(z)$ is a flat $\mathbf{Z}$-algebra we get $\varphi$ flat using the Local Criteria of Flatness [4, Theorem 49, (20.C)].

Induct on $m$ and assume $m>1$. Then $B /\left(z_{m}\right)$ is a flat $\mathfrak{N} /\left(\Gamma_{m}\right)$-algebra by induction hypothesis. As in the case $m=1$ we see that $\operatorname{Tor}_{1}^{\mathfrak{N}}\left(\mathfrak{N} /\left(\Gamma_{m}\right), B\right)=0$ and so $\varphi$ is flat using the Local Criteria of Flatness.

Remark 17. Let $R$ be a regular local ring, $s=\left(s_{1}, \ldots, s_{m}\right) \in \mathbf{N}^{m}$ and $z=$ $\left(z_{1}, \ldots, z_{m}\right)$ be a part of a regular system of parameters of $R$. Then $B=R /\left(z_{1}^{s_{1}}, \ldots, z_{m}^{s_{m}}\right)$ satisfies the above lemma.

Using the above lemma and Theorem 14 we get the following result.

Theorem 18. In the notation and hypothesis of the above lemma, $B$ is a filtered inductive limit of some Noetherian local $\mathbf{Z}$-algebras $\left(F_{i}\right)_{i}$ essentially of finite type with $F_{i} / \sqrt{(0)}$ regular local rings.

As in Corollary 8 we get the following result.

Corollary 19. In the notation and hypothesis of Lemma 16 , suppose that $B /(z)$ is essentially of finite type over $\mathbf{Z}$. Then $B$ is essentially smooth over a local $\mathfrak{N}$-algebra $D$ of type $C /(p-b)$, where $(C, \mathfrak{q})$ is a local ring, esentially smooth over $\mathfrak{N}$ with $b \in \mathfrak{q}^{2}$ (so $D / \Gamma D$ and $C / \Gamma C$ are regular).

Theorem 20. Let $(A, \mathfrak{m}, k)$ be a Noetherian local ring, $T=\left(T_{1}, \ldots, T_{n}\right)$ some variables and $\mathbf{b}$ a nilpotent $i d e a l$ of $A$. Suppose that $R=A / \mathbf{b}$ is a regular local ring and the $B Q$ Conjecture holds for $R$ (for example $p:=$ char $k=0$, or $p \notin \mathfrak{m}^{2}$ ). Then any finitely generated projective $A[T]$-module is free.

Proof. Let $M$ be a finitely generated projective $A[T]$-module. Then $\bar{M}=M / \mathbf{b} M$ is finitely generated projective over $R[T]$ and so it is free. Let $x=\left(x_{1}, \ldots, x_{r}\right)$ be a system of elements from $M$ inducing a basis in $\bar{M}$. Then $M=<x>+\mathbf{b} M=$ $<x>+\mathbf{b}^{t} M=\left\langle x>\right.$ for $t \in \mathbf{N}$ with $\mathbf{b}^{t}=0$. Thus the map $\varphi: A[T]^{n} \rightarrow M$ given by $\left(a_{1}, \ldots, a_{n}\right) \mapsto \sum_{i} a_{i} x_{i}$ is surjective. Set $N=\operatorname{Ker} \varphi$. Tensorizing with $A[T] /(\mathbf{b})$ the exact sequence

$$
0 \rightarrow N \rightarrow A[T]^{n} \rightarrow M \rightarrow 0
$$

we get the following exact sequence

$$
\operatorname{Tor}_{1}^{A[T]}(M, A[T] /(\mathbf{b})) \rightarrow N / \mathbf{b} N \rightarrow R[T]^{n} \rightarrow \bar{M} \rightarrow 0,
$$

where the first module is zero because $M$ is a flat $A[T]$-module. As the last map is injective we see that $N=\mathbf{b} N=\mathbf{b}^{t} N=0$, that is $\varphi$ is an isomorphism.

\section{REFERENCES}

[1] H. Bass, Some problems in "classical" algebraic K-theory in Algebraic $K$-theory II, Springer Lect. Notes 342, (1973), 1-70.

[2] T. Y. Lam, Serre's Conjecture, Springer Lect. Notes in Math., 635, Berlin, 1978.

[3] H. Lindel, On the Bass-Quillen Conjecture concerning projective modules over polynomial rings, Invent. Math., 65, (1981), 319-323. 
[4] H. Matsumura, Commutative Algebra, Benjamin, New-York, 1980.

[5] M. P. Murty, Projective A[X]-modules, J. London. Math. Soc., 41, (1966), 453456.

[6] M. P. Murty, A letter containing Swan's notes on Lindel's results, 1987.

[7] D. Popescu, Polynomial rings and their projective module, Nagoya Math. J., 113, (1989), 121-128.

[8] D. Popescu, On a question of Swan. With an appendix by Kestutis Cesnavicius, Algebraic Geometry, 6(6), (2019), 716-729, arXiv/AC:1803.06956.

[9] D. Quillen, Projective modules over polynomial rings, Invent. Math., 36, (1976), 167-171.

[10] R. Rao, Two Examples of the Bass-Quillen-Suslin Conjectures, Math. Ann., 279, (1987), 227-238.

[11] R. Rao, The Bass-Quillen conjecture in dimension three but characteristic $\neq 2,3$ via a question of A. Suslin, Invent. Math., 93, (1988), 609-618.

[12] M. Roitman, On projective modules over polynomial rings, J. Algebra, 58, (1979), 51-63.

[13] A. A. Suslin, Projective modules over a polynomial ring are free, Soviet. Math. Dokl., 17, 1976, 1160-1164.

[14] R. Swan, Neron-Popescu desingularization, in "Algebra and Geometry", Ed. M. Kang, International Press, Cambridge, (1998), 135-192.

Dorin Popescu, Simion Stollow Institute of Mathematics of the Romanian Academy, Research unit 5, University of Bucharest, P.O.Box 1-764, Bucharest 014700, ROMANIA

E-mail address: dorin.popescu@imar.ro 